\title{
Bilateral Blindness Due to Left Fronto-Temporal Lobe Glioblastoma Multiforme
}

\author{
Thapa BB ${ }^{1}$, Neupane $\mathrm{J}^{2}$, Moktan $\mathrm{S}^{3}$
}

\section{ABSTRACT}

Introduction: Glioblastoma multiforme is the most common primary malignant brain tumor, with a very poor prognosis. Direct compression of any structure in the visual pathway or chronic papilloedema is the cause of loss of vision in these patients. Case Report: 30 years old male from Dailekh was brought by his elder brother with the complaint of complete vision loss in both eyes for 45 days. He also had hearing difficulty on right side, headache, right sided weakness and abnormal body movement. His Visual acuity was no perception of Light in both eyes. Pupil was mid-dilated and not reacting to light. Fundus examination revealed bilateral Pallid disc edema. MRI brain revealed left fronto-temporal GBM compressing optic chiasm. Neurosurgical consultation was obtained and counseling was done regarding disease and its treatment. Due to poor prognosis and poverty patient choose palliative conservative treatment. Conclusion: This case highlights the possibility of bilateral Blindness in unilateral GBM. GBM has very poor outcome even after treatment.

Keywords: Blindness, Glioblastoma multiforme, Magnetic resonance imaging

1. Dr. Bikram Bahadur Thapa

2. Dr. Joshan Neupane

3. Dr. Suman Moktan

${ }^{1}$ Department of Ophthalmology, Nepalgunj Medical College and Teaching Hospital, Nepalgunj, Banke ${ }^{2}$ Department of Neuosurgery, Nepalgunj Medical College and Teaching Hospital, Nepalgunj, Banke

${ }^{3}$ Nepalgunj Medical College and Teaching Hospital, Nepalgunj, Banke

\section{Address for Correspondance:}

\author{
Dr. Bikram Bahadur Thapa \\ Department Of Ophthalmology \\ Nepalgunj Medical College and Teaching Hospital \\ Nepalgunj, Banke, Nepal \\ Email: drbbthapa@gmail.com
}

\section{INTRODUCTION}

Glioblastoma multiforme (GBM) is the most common and lethal malignant primary brain tumor, with an incidence of $3.19 / 100,000$ person years and remarkably poor prognosis showing a 5-year survival rate of $4-5 \% .{ }^{1}$ GBM occurs most commonly in sub cortical white matter of temporal, parietal, frontal and occipital lobe. Combined fronto-temporal is typical location whereas brainstem, cerebellum and spinal cord are less common site. ${ }^{2}$ Intra-cranial Space occupying lesions (ICSOL) may be responsible for a range of neuro-ophthalmic deficits including visual problems, optic disc pallor or oedema, and oculomotor disturbances. Lesions may either affect vision directly by compression or infiltration anywhere along the visual pathways or visual cortex or indirectly by prolonged raised intracranial pressure (ICP) causing papilloedema. ${ }^{3}$

The common causes of bilateral blindness are cataract, pathological myopia, age related macular degeneration, glaucoma, optic neuropathy, diabetic retinopathy and corneal opacity. ${ }^{4}$

Bilateral occipital lobe ischemia or infarction or hemorrhage, bilateral occipital lobe trauma, chronic Idiopathic Intracranial
Hypertension, chronic papilloedema, chiasmal compression, cavernous sinus thrombosis and methyl alcohol poisoning etc are less common causes of bilateral blindness. ${ }^{4-6}$ The cause of bilateral blindness in GBM is either direct chiasmal compression or chronic papilloedema. There are reports of partial blindness from GBM at different stage of treatment from different part of world ${ }^{1-3}$ but in this case we report bilateral complete blindness as a presentation of GBM in a Nepalese patient.

\section{CASE REPORT}

A 30 years old male patient from Dailekh District was brought by his elder brother in ophthalmology outpatient department of Nepalgunj Medical College, Nepalgunj with the complaint of complete vision loss in both eyes for 45 days. It was painless and progressive in natures. He had a history of hearing difficulty on right side for 3 months. He experienced multiple episodes of headache for 1 year, which was gradual on onset, mild to moderate intensity, mostly in frontal region. He also had history of weakness in right side of body for 1 month. He had multiple episodes of abnormal body movement from last 15 months, which might be the initial symptom of the diseases but due to poverty and religious values he did not able to consult heath 
care provider. In his village people believe abnormal body movement was caused by sin, effect of ghost and as effect of god on him. Thus, He was taken to Dhami and Jhakri for treatment for multiple times with the hope of recovery.

On ophthalmic evaluation his Visual acuity (VA) was no perception of light (NPL) in both eyes. Intraocular pressure (IOP) was $16 \mathrm{mmHg}$ on Goldman applanation tonometer (GAT) in both eyes. Anterior segment evaluation including cornea and lens revealed no abnormalities. Both pupil were fixed and dilated not reacting to light. On fundus examination both optic discs were elevated with blurred margin, obliterated cup, disc hemorrhage, pallid color and dilated venules suggesting chronic papilloedema as seen in Disc photo (Figure 1a and 1b) which was also seen in MRI (Figure 3). Rest of the fundus reveals no abnormality. Color vision and visual field test could not be performed.

Magnetic resonance Imaging (MRI) brain was done, which revealed heterogenous lesion of $90 \times 70 \times 65 \mathrm{~mm}$ in left cerebral hemisphere involving left fronto-temporal lobe, parietal lobe and left basal ganglia (Figure $\mathbf{2 a}$ and $\mathbf{2 b}$ ). The lesion is also extending to suprasellar cistern with compressing optic chiasm and pituitary gland (Figure $2 \mathbf{c}$ ). Lesion showed enhancement after intra-venous Gadolinium with cystic and necrotic changes. Mass effect evidenced by effacement of adjacent sulci and ipsilateral lateral ventricle with resultant midline shift (Figure 2c). Magnetic Resonance spectroscopy (MRS) of the lesion showed a raised choline peak with a depressed N-acetyl aspartate peak with reversed Hunter's angle confirming diagnosis of Glioblastoma Multiforme.

Pure tone audiometry (PTA) revealed total hearing loss in right side and mild sensori-neural hearing loss in left side (Figure 4). Neurosurgical consultation was obtained. After detailed evaluation and explaining surgical outcome, need of postoperative care, cost of treatment and prognosis by consultant Neurosurgeon, patient denied surgical treatment and given Intracranial pressure(ICP) lowering agent and analgesics.

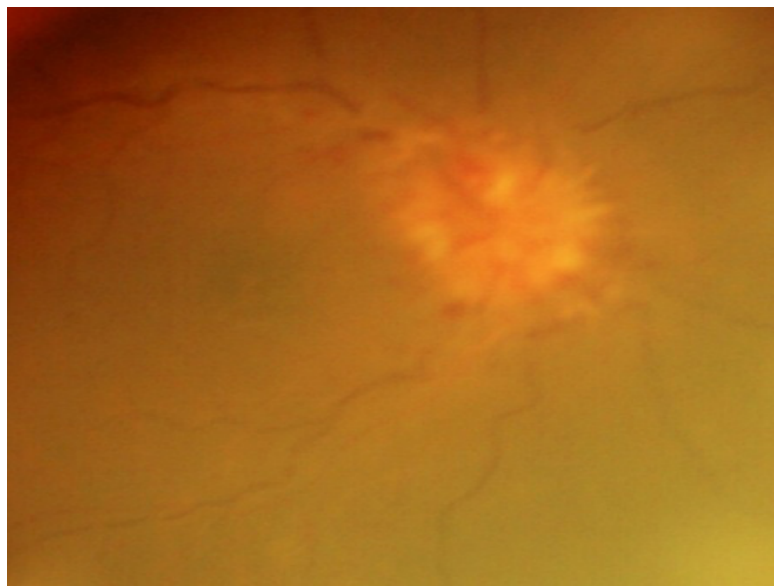

Figure 1a: Disc photo of right eye showing established chronic disc edema

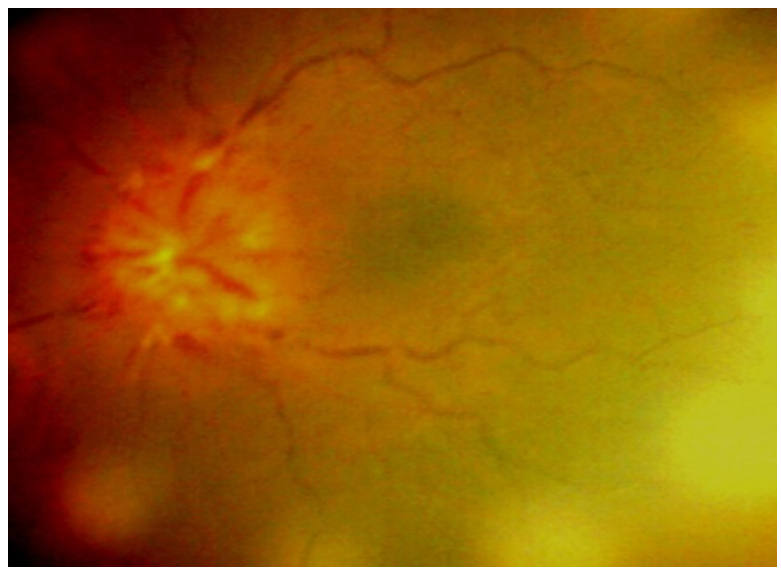

Figure $1 b$ : Disc photo of left eye showing established chronic disc edema

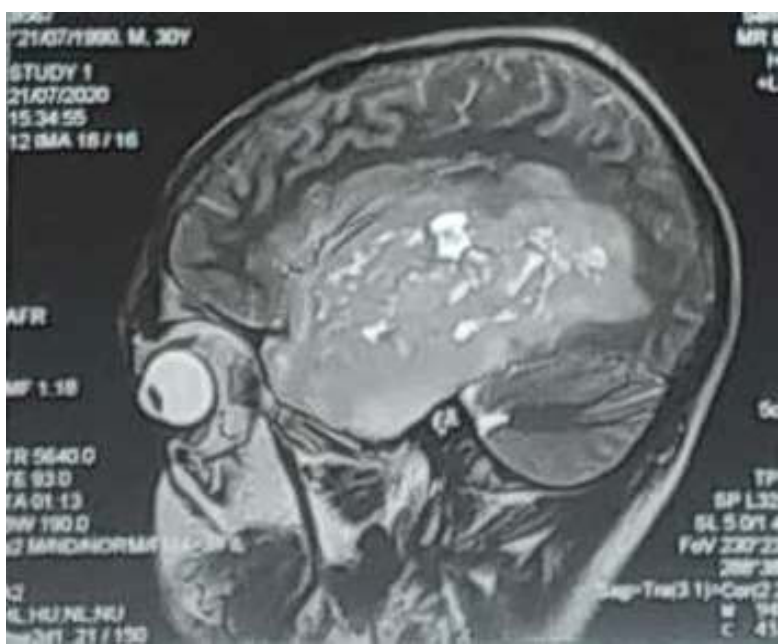

Figure 2a : Sagittal section of 72 -Weighted scan showing mass located in left fronto-temporal lobe with extension in parietal lobe and basal ganglia

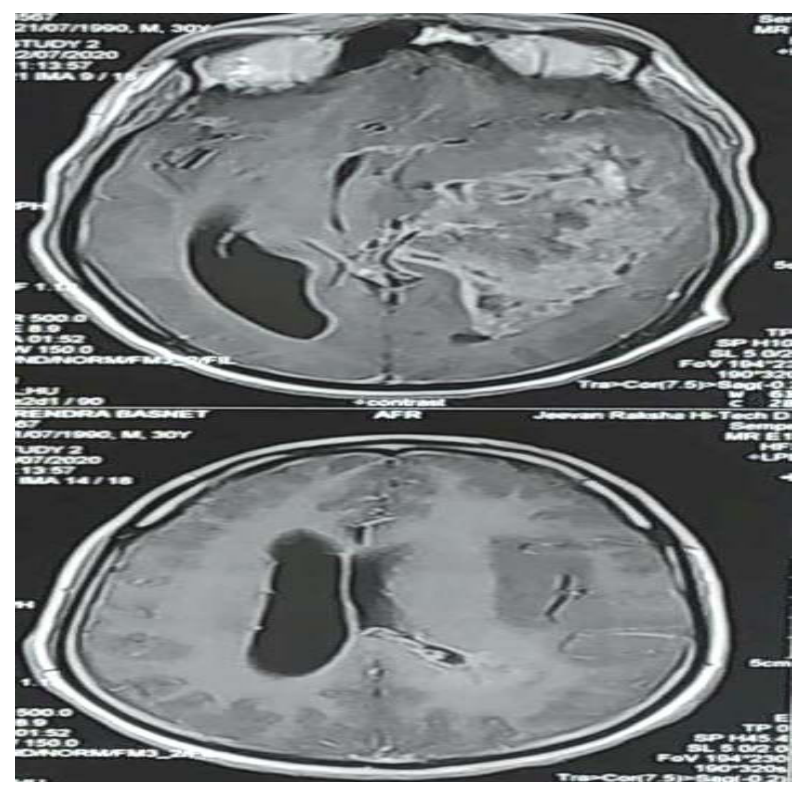

Figure $2 b$ : Axial section of T1-W scan showing mass on left temporal lobe with midline shift, enlarged contra-lateral lateral ventricle and effacement of adjacent sulci 


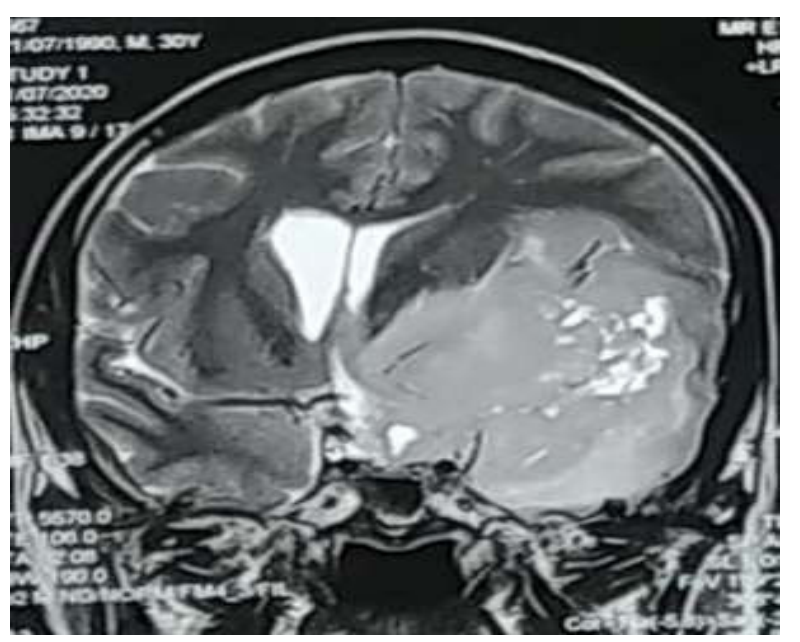

Figure $2 c$ : Coronal section of $T 2-W$ scan showing heterogeneous mass in left fronto-temporal lobe compressing optic chiasm with midline shift and enlarged contra-lateral ventricle

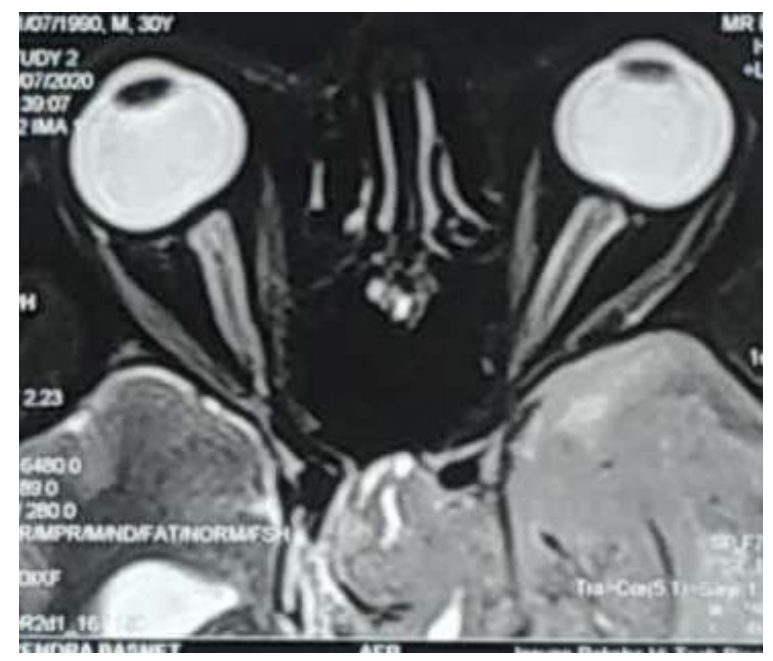

Figure 3 : T2 weighted MRI Axial scan showing enlarged perioptic subarachnoid space with thinned out optic nerve

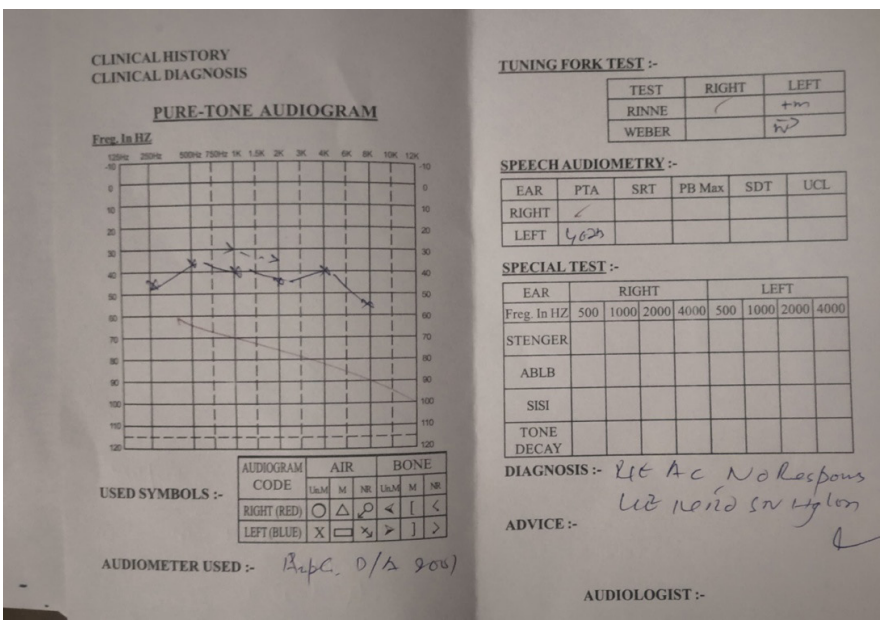

Figure 4 : Pure Tone Audiomety revealing total hearing loss in right side and mild sensorineural hearing loss in left side

\section{DISCUSSION}

Glioblastoma multiforme associated with symptoms due to mass effect and disturbance of local brain function. Visual problems are the neuro- ophthalmic deficits observed in these patients. Lesions may affect vision directly by compression or infiltration anywhere along the visual pathways or visual cortex or indirectly as a result of the optic atrophy caused by chronic papilloedema. ${ }^{3}$ In the lesion of the anterior visual pathways and chronic papilloedema, the pupillary response to light is attenuated and the optic disc appears abnormal.

Delay in the presentation to the health care provider is a known problem in Nepal due to difficult geography, lack of transportation facility, poverty, and religious value, belief in local traditional healer including Dhami and Jhakri especially in Far-western and Midwestern Hills. People seek health service only when they need help of another person to carry out their basic daily activities. He neglected all problem because of hearing difficulty, weakness of body, occasional headache and occasional abnormal body movements. ${ }^{8}$ This indicates that the most significant neurological deficit caused by his brain tumor was the complete loss of his sight as shown by Seth et al in his case report ${ }^{2}$. Thus strong and dedicated governmental promotional, preventive and curative health system is needed to fight against these problems. Direct compression of the optic chiasm need to be considered to account for the bilateral complete vision loss in this case as MRI showed chiasmal compression by tumor. An acute "spike" superimposed on his chronically raised ICP causing decompensation of the swollen optic disc can be another mechanism for visual loss in this case. This fact was also highlighted by Seth et al. ${ }^{2}$

Current treatments cannot cure GBM patients and help to extend their survival only. Surgical resection followed by radiotherapy and chemotherapy with temozolamide is the preferred treatment of $\mathrm{GBM}^{1}$. Intracranial pressure lowering agent like Acetazolamide, corticosteroids to reduce perilesional edema and analgesics to relieve pain are used in palliative care as done in this case. ${ }^{1,2}$ The median survival of the patient is around 15 months with the treatment. Counseling regarding these facts including nature of disease, treatment options, treatment outcomes and prognosis help patient and their family to make best cost effective and rational choice.

\section{CONCLUSION}

This case highlights the risk of irreversible visual loss in patients with brain tumors, when the lesion is compressing the visual pathways. In developing country like Nepal, patients present to the health care service once disease is advanced, thus awareness program should be increased up to each community and school. GBM is lethal brain tumor, having 5 year survival rate of $4-5 \%$. So cost- effective approach and affective counseling is vital in its management. 


\section{REFERENCES}

1. Batash R, Asna N, Schaffer P, Francis N, Schaffer M. Glioblastoma Multiforme, Diagnosis and Treatment; Recent Literature Review. Current Medicinal Chemistry 2017; 24:3002-9.

2. Seth A, Short S, Bremner F. Sub-acute blindness in a patient with a temporal lobe astrocytoma. BMJ Case Rep 2009; doi: 10.1136/ bcr.01.2009.1513, PMID: 21691404

3. Vloeberghs $M$. Decision making in paediatric brain tumours: a neurosurgical perspective. Current Pediatrics 2005; 15: 406-11

4. Sainz-Gómez C, Fernández-Robredo P, Salinas-Alamán Á, Montañés J. M, Berasategui J. M. E. et al. Prevalence and causes of bilateral blindness and visual impairment among institutionalized elderly people in Pamplona, Spain. Eur J Ophthalmol 2010; 20: 442-450

5. Kakaletsis N, Tziomalos K, Spilioti M, Diavati O, Chryssogonidis I, et al. Bilateral Cortical Blindness due to Bilateral Occipital Infarcts without Anosognosia. J Neurol Stroke 2016; 4: 00120. DOI: 10.15406/jnsk.2016.04.00120

6. Verma R, Gupta M, Chaudhari TS. Neurogenic vision loss: Causes and outcome. An experience from a tertiary center in Northern India. J Neurosci Rural Pract 2014; 5:340-8.

7. Shrestha P, Pant B. Glioblastoma multiforme or brain abscess. Journal of Institute of Medicine Nepal 2009; 31: 23-25.

8. Thapa BB, Gurung S, Singh S, Aryal M. Early phase Vogt Kayanagi Harada disease in elderly Nepalese women. Nep J Ophthalmol 2020; 12: 146-152. 